\title{
Structure of the sucrose-specific porin ScrY from Salmonella typhimurium and its complex with sucrose
}

\author{
Doris Forst' ${ }^{1}$, Wolfram Welte ${ }^{2}$, Thomas Wacker ${ }^{1}$ and Kay Diederichs ${ }^{2}$
}

\begin{abstract}
The X-ray structure of a sucrose-specific porin (ScrY) from Salmonella typhimurium has been determined by multiple isomorphous replacement at $2.4 \AA$ resolution both in its uncomplexed form and with bound sucrose. ScrY is a noncrystallographic trimer of identical subunits, each with $\mathbf{4 1 3}$ structurally well-defined amino acids. A monomer is built up of 18 anti-parallel $\beta$-strands surrounding a hydrophilic pore, with a topology closely similar to that of maltoporin. Two non-overlapping sucrose-binding sites were identified in difference Fourier maps. The higher permeability for sucrose of ScrY as compared to maltoporin is mainly accounted for by differences in their pore-lining residues.
\end{abstract}

Gram-negative bacteria protect their vulnerable cytoplasmic membrane by a peptidoglycan layer and an outer membrane $(\mathrm{OM})^{1,2}$. It has been estimated that $\sim 10 \%$ of all genes in $E$. coli are involved in transport of small nutrient molecules across the $\mathrm{OM}$, the periplasmic space with the peptidoglycan and the cytoplasmic membrane (CM) ${ }^{3}$. Sugar-specific, active transport systems in the CM with $K_{\mathrm{m}}$ values in the micromolar range maintain a low concentration of monosaccharides, disaccharides and unbranched oligosaccharides in the periplasm ${ }^{4,5}$. In contrast, sugar transport across the OM is purely passive, driven by diffusion. According to Fick's equation the flux of a species of molecules accross the OM is proportional to the concentration difference between the external space and the periplasmic space. The high value of the proportionality constant, the permeability, is due to the porins, the major protein component of the OM. So-called 'general diffusion porins' 6,7 or 'nonspecific porins' form water-filled channels through which ions and polar molecules, smaller than the exclusion limit of the channel, may pass. Nonspecific porins are between $30,000-50,000 M_{\mathrm{r}}$ and form trimers. The ionic permeability of general diffusion porins can be measured as current flowing through porins reconstituted into black lipid membranes ${ }^{6}$ or through small OM fragments in patch-clamp experiments ${ }^{8}$. Plots of current against ion concentration are linear, showing that ions pass through the $\mathrm{OM}$ in a diffusion-like process. The linear relation of current and ion concentration and the differences in the permeability of anions and cations can be rationalized qualitatively by theoretical models?.

The structures of five general diffusion porins have been determined by $\mathrm{X}$-ray crystallography ${ }^{10-13}$. These structures are variations of a 16-stranded antiparallel $\boldsymbol{\beta}$-barrel with nearest-neighbor connections between strands. The connections form loops (L) of varying length on the external side and mostly hair-pin turns ( $T$ ) on the periplasmic side of the OM. The third external loop (L3) folds along the inner barrel wall and constricts its lumen in the center of the membrane, giving the channel an hourglass-like cross section that allows for a high, but charge-dependent permeability ${ }^{14}$.
The permeation of solute molecules through the channel can be treated with reaction-rate theories, either transition state theory ${ }^{15}$ or Kramers theory ${ }^{16}$. The latter appears to be more appropriate to aqueous protein-substrate systems. The rate coefficient for permeation contains a Boltzmann factor $\exp \left[-\Delta G^{*} / R T\right]$ with the activation free-energy $\Delta \mathrm{G}^{*}=\Delta \mathrm{H}^{*}-\mathrm{T} \Delta \mathrm{S}^{*}$ of the entry process (formula (14) in ref. 16). The entropy term $-\mathrm{T} \Delta \mathrm{S}^{*}$ will represent a considerable fraction of the free-energy barrier, as the permeating molecules are confined to the narrow cross section of the channel.

Under growth-limitation conditions the cells must strive to increase the permeability further to yield a sufficient flux of nutrient molecules at shallow concentration gradients. Increasing the density of nonspecific porins in the $\mathrm{OM}$ and increasing the cross section of the porin channel are inappropriate means because the $\mathrm{OM}$ is already densely packed with porins ${ }^{17}$ and because an increased exclusion limit would weaken the protective effect of the $\mathrm{OM}$. Another means to increase the permeablity is to decrease the free energy barrier for entrance into the channel so that the rate of entrance is increased. This can be effected by introducing binding sites specific for certain nutrient molecules, such as sugars or nucleosides, since the negative contribution of the enthalpy of one or several binding sites will decrease $\Delta \mathrm{G}^{*}$. Binding sites arranged along the inner channel wall thus will increase the permeability compared to the case of a nonspecific channel, as long as the concentrations of the substrate molecules are well below their dissociation constants $\left(K_{d}\right)$.

Indeed, porins specific for different small nutrient molecules are expressed by Gram-negative bacteria under conditions of growth limitation. One example is maltoporin (also called LamB due to its role as a receptor for phage $\lambda$ ) which is specific for maltose and maltooligosaccharides. It is coded on the mal regulon together with a maltose transport complex of the ATP binding cassette (ABC) type and a periplasmic binding protein ${ }^{18}$. Maltoporin is expressed at disaccharide concentrations lower than $\sim 100 \mu \mathrm{M}^{19}$, while the $K_{d} \mathrm{~s}$ of the binding sites for disaccharides of both glycoporins are in the $10 \mathrm{mM}$ range. The $K_{d} s$ drop for oligosaccharides with 3, 4 and 5 glucosyl units but remain constant at a value of $\sim 300 \mu \mathrm{M}^{20}$ for longer ones.

'Institut für Biophysik und Strahlenbiologie, Albert-Ludwigs-Universität, Albertstr. 23, 79104 Freiburg im Breisgau, Germany. ${ }^{2}$ Fakultät für Biologie, Universität Konstanz, Box M656, 78457 Konstanz, Germany. 


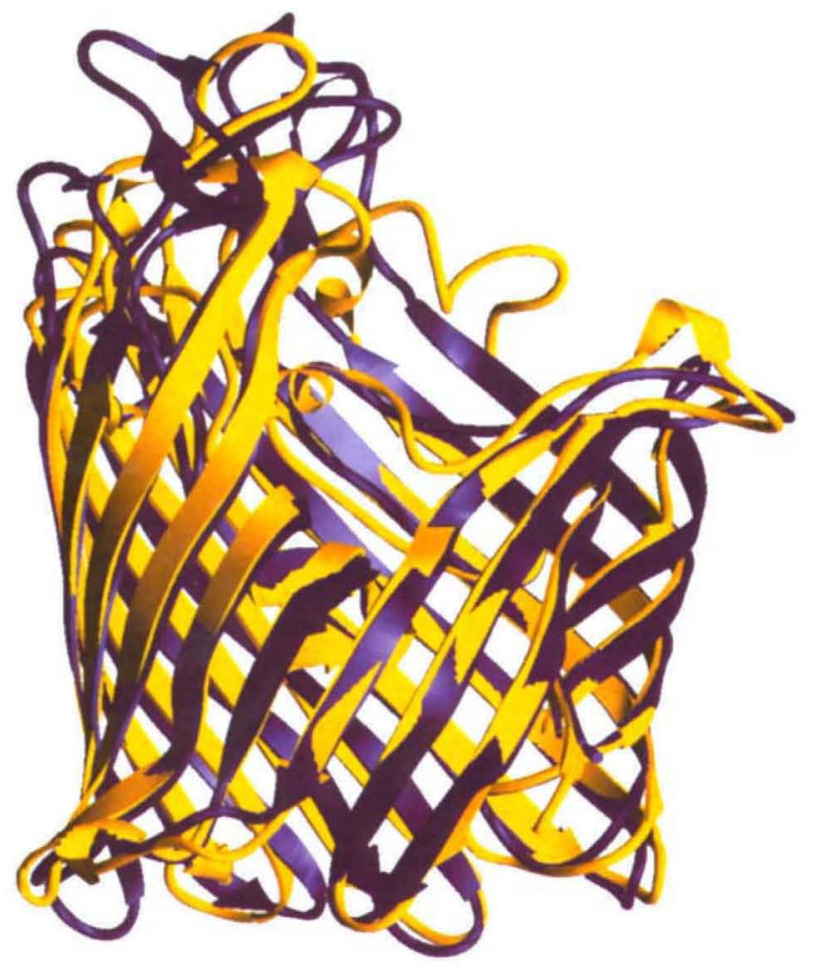

The structure of maltoporin with and without bound maltooligosaccharides and also with sucrose has been determined by $\mathrm{X}$-ray crystallography ${ }^{21-24}$. Maltoporin possesses the same overall structure as the general diffusion porins, except that its barrel contains 18 strands. As expected from binding experiments ${ }^{20}$, maltoporin possesses a chain of 3-5 low affinity binding sites for glucosyl groups extending along the wall of the channel.

Schmidt et al..$^{25}$ have discovered a sucrose uptake system on plasmid pUR400 in Salmonella typhimurium and Escherichia coli that confers to the cells, under growth limitation conditions, the ability to grow on sucrose as a sole carbon source. The scr regulon codes for a sucrose-specific phosphoenoltransferase-dependent transport (PTS) complex in the cytoplasmic
Fig. 1 Ribbon superposition of ScrY (yellow) and maltoporin (blue) with the periplasmic side and the external side at the bottom and top, respec tively. The barrel consists of 18 antiparallel strands and has a kidneyshaped crossection of between $30 \AA$ and $50 \AA$ diameter. The height varies between $20 \AA$ near the trimer axis and $50 \AA$ at the trimer periphery. The tilt angles of the $\beta$-strands relative to the trimer axis is between $35^{\circ}$ and $55^{\circ}$, and the barrel shear number ${ }^{73}$ amounts to +22 . This figure was prepared with RIBBON574.

membrane. It was later found that one gene, scrY, codes for a porin that is specific for sucrose and maltooligosaccharides and that the scr regulon is also found in the genome of Klebsiella pneumoniae 26,27 .

$\mathrm{N}$-terminal sequencing ${ }^{28}$ showed that mature ScrY and maltoporin possess 483 and 421 residues respectively. Upon comparing sequences it was found that a C-terminal ScrY domain of 411 residues can be aligned with the Maltoporin sequence with an overall identity of around $20 \%{ }^{26,27}$. The N-terminal 72 residues of ScrY have no equivalent in maltoporin.

We have crystallized ScrY $\mathrm{Y}^{29}$ and solved by multiple isomorphous replacement (m.i.r.) the structure of the pore domain (residues $71-483$ ) at $2.4 \AA$ resolution both with and without bound sucrose.

\section{Crystallographic problems and solutions}

Three presumably interrelated problems rendered the structure determination of ScrY difficult. First, the space group reported for the low-resolution data ${ }^{29}$ of the preliminary crystallographic investigation proved irreproducible. At resolution of $4 \AA$ and beyond, additional reflections $(-h+k+l \neq 3 n)$ were found between the reflections of the obverse-indexed hexagonal-rhombohedric lattice $(-h+k+1=3 n)$, turning the lattice into a hexagonal-primitive one. These reflections were of negligible intensity at low resolution $(6 \AA)$, but were of the same average intensity as those of the $\mathrm{R} 3$ lattice at resolution better than $3 \AA$, overall increasing the proportion of weak reflections. This finding can be explained by a slight distortion of the R3 space group, leading to the $\mathrm{P} 3_{(1,2)}$ subgroup with a trimer in the asymmetric unit, whose non-crystallographic symmetry axis is close to, but does not coincide with, the rotation axis of the R3 system. The three-fold rotational symmetry of the

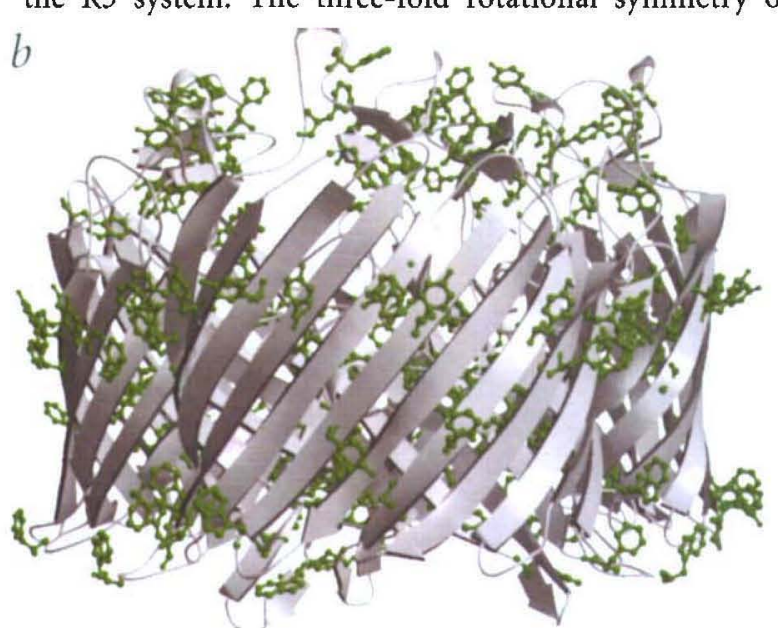

Fig. 2 View of the membrane spanning surfaces of the glycoporin trimers. $\mathbf{a}, \mathbf{S} \boldsymbol{\text { crY; }} \boldsymbol{b}$, maltoporin. Aromatic residues are added to the ribbon representation of both porins. The two girdles of aromatic residues that bound the hydrophobic zone of $30 \AA$ width are discernible. In ScrY they are less regular and some aromatic residues are within the hydrophobic zone. Moreover, maltoporin differs from ScrY by an additional clustering of aromatic residues in the external loops. This figure was prepared with Molscript ${ }^{75}$ and Raster3D ${ }^{76}$. 


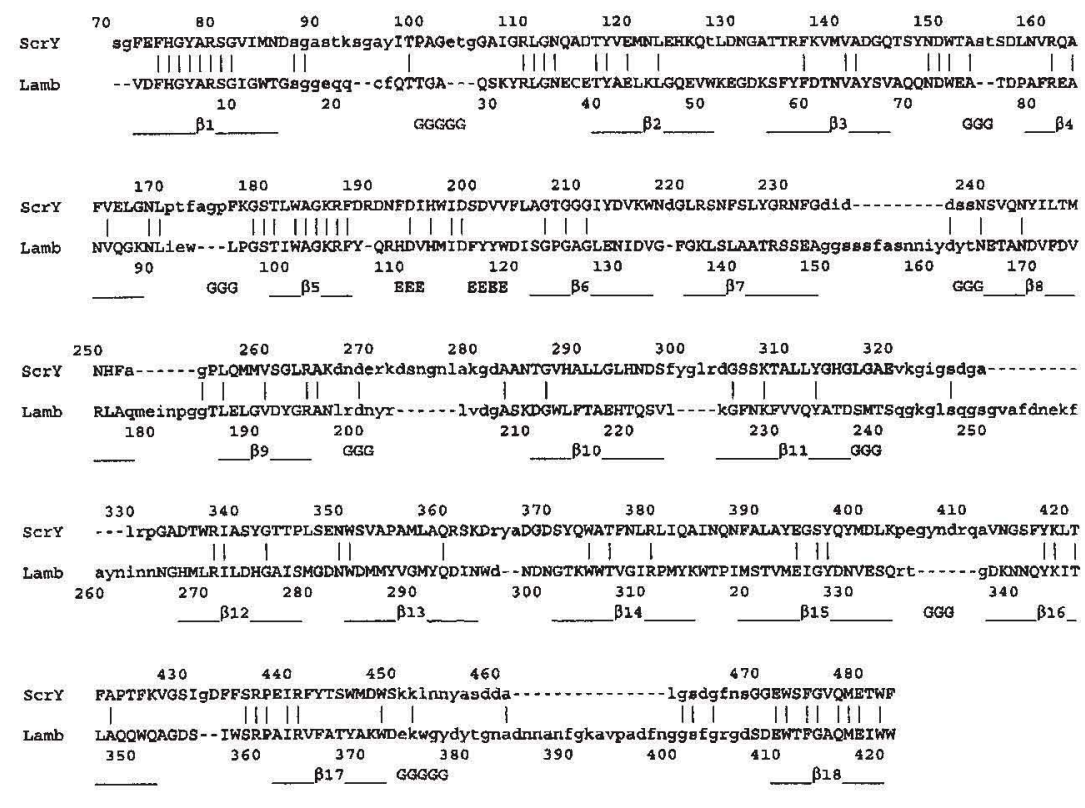

hexagonal primitive lattice was well fulfilled, and the absence of intensites of the $(0,0,1)$ reflections with $l \neq 3 n$ confirmed the space group $\mathrm{P}_{(1,2)}$.

Second, we found the ScrY crystals to be highly anisomorphous, with crystals even from the same crystallization setup exhibiting variations of unit cell axes in the order of $2-3 \%$, leading to difficulties in interpretation of difference Patterson and difference Fourier syntheses. $R$-factors between native crystals ranged mostly between $20-30 \%$. Therefore, complete data sets had to be collected on single crystals.

Third, ScrY crystals were twinned, with twinning fraction $\alpha$ evenly distributed in the range $0-0.5$. Procedures as described in the Methods section were used to determine the twinning fraction according to equation 1 and for detwinning the data according to equation 2.

Despite these difficulties initial single isomorphous replacement phases were obtained by solving the difference Patterson map of the cocrystallized $\mathrm{KAu}(\mathrm{CN})_{2}$-derivative. Interpretation of other derivatives followed (Table 1). It was found that detwinning provided cleaner difference Patterson functions and was necessary for their interpretation. However, for heavyatom refinement and phasing the observed (and not the detwinned) amplitudes were used, as detwinning produces datasets of lower completeness, and we were able to identify derivative datasets with low twinning fractions. M.i.r phasing allowed us to obtain an interpretable density map at $2.9 \AA$ resolution, into which residues $71-483$ of the mature sequence could be built in several macrocycles of X-PLOR refinement and manual improvements of the model, using $\mathrm{O}$.

After completing the final X-PLOR refinements, SHELXI refinements of the native and sucrose-complexed models confirmed the twinning fraction as determined by equation 1 and showed that inclusion of $\alpha$ as a refinable parameter (as opposed to no twinning treatment) decreased both $R_{\text {free }}$ and $R_{\text {crys }}$ by only $0.12 \%$ and $0.20 \%$ respectively. However, results of these refinements were discarded since - in addition to requiring almost prohibitive computing resources - it was not possible to restrain the positions of NCS-related water molecules in a simple way. Therefore, final statistics (Table 2) except for twinning fractions are based on X-PLOR refinement.
Fig. 3 Structural alignment between ScrY and E. coli maltoporin. Capital letters denote structurally equivalent residues based on a 3.8 A cutoff, whereas residues without a structural counterpart are shown in lower case letters. In addition to the 18 strands of the main $\beta$-barrel, short pieces of regular secondary structure $(E \beta$ strand; G, $3_{10}$-helix) as classified by DSSP77 are also given.

\section{Description of the structure}

The model of the $\mathrm{C}$-terminal 413 residues of ScrY represents the complete transmembrane domain. As in all porins whose structures have been analyzed so far, ScrY is basically a $\beta$-barrel with nearest neighbor connections between strands and with large loops (L) of irregular length exposed to the external surface. $\mathrm{L} 3$ is attached by polar and apolar interactions to the inner barrel wall. It constricts the channel cross section near the center to $8 \times 11 \AA$ so that the channel lumen possesses an hour-glass shaped longitudinal cross section with a large external and a narrower periplasmic vestibule.

Like other porins, ScrY forms stable trimers in the outer membrane. In contrast to the structures of the nonspecific porins, which all consist of $16 \beta$-strands, the two glycoporins ScrY and maltoporin ${ }^{21}$ possess 18 strands. The observed barrel structure has a kidney-shaped cross section with dimensions of roughly $50 \AA \times 28 \AA$ and a height of between $25 \AA$ near the trimer center and $50 \AA$ at the trimer periphery.

When the MIR structure analysis of ScrY was begun, the low sequence similarity with maltoporin led us to expect considerable structural differences between the two porins. However, the two independently solved structures show a surprisingly large degree of structural conservation (Fig. 1). The gross differences between both glycoporins are in the external loops which tend to tilt over the pore vestibule in both porins like petals and thereby form a constriction at the entry to the common vestibule of all three monomers. The residues of the phage- $\lambda$ binding site of maltoporin on L9, L4, L6 are not conserved in ScrY. L9 contains a short $3_{10}$-helix and thereafter a $\mathrm{Ca}^{2+}$-binding loop segment. In the latter, the ion is bound in a distorted tetragonal-bipyramidal geometry. The longest loop L1, which consists of 29 amino acids and contains a short $3_{10}$-helix, folds down towards the constriction site and is involved in hydrogen bonds with L3. These hydrogen bonds stabilize the structure of both loops. Generally, ScrY is less 'closed' than maltoporin as L4, L6, L8 and L9 are shorter. The similarity of the backbones of both glycoporins is quite high in view of the modest sequence homology: using a 3.8 A cutoff, 315 of the $413 \mathrm{C} \alpha$ atoms of ScrY can be superimposed with those of maltoporin with a root-mean-square (r.m.s.) deviation of only $1.3 \AA$.

Among the periplasmic turns ( $\mathrm{T}$ ), $\mathrm{T} 5$ is larger in ScrY and protrudes in a radial direction from the barrel, whereas T4 is markedly shorter in ScrY. T5 is involved in the crystal-contacts and $\mathrm{T} 8$ folds into the channel mouth.

\section{The monomer-monomer interface}

Trimer formation is stabilized through many polar interactions between monomers at the top and bottom of the $\beta$-barrel. In between, there exists an apolar region formed by small 
$a$

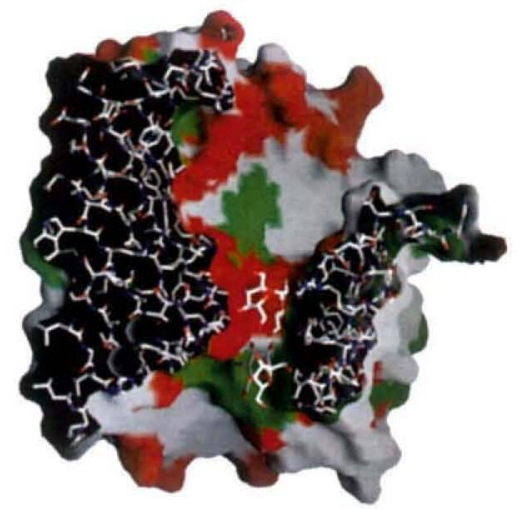

$b$

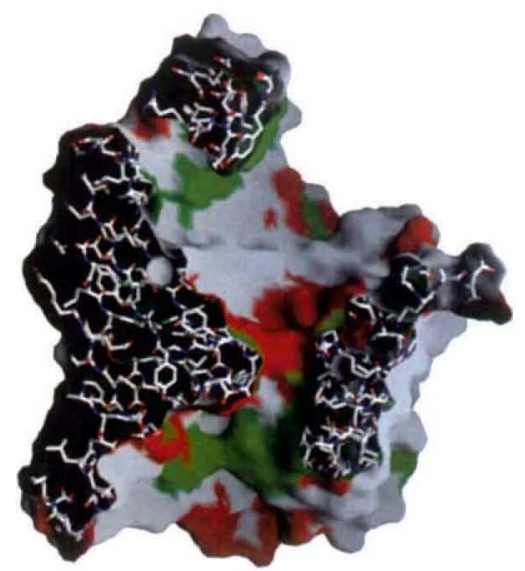

Fig. 4 Images $^{78}$ comparing the hourglassshaped inner channel surfaces of a, ScrY complexed with two sucrose molecules and $b$. maltoporin. The cutting plane is spanned by the trimer axis and a radial beam connecting the axis and the constriction site center. The molecular surface near Asp, Glu, Arg, Lys is colored red whereas near Phe, Tyr, Trp it is colored green.

chain of binding sites or a binding slide for maltose and maltoligosaccharides. In structures of maltoporin complexed with maltooligosaccharides, most of the glucosyl groups are in van der Waals contact with some of the aromatic residues.

In ScrY, the four external of the six aromatic residues found in maltoporin are

hydrophobic amino acids. The low average B-factor of around 18 $\AA^{2}$ indicates the rigidity of this region. On the periplasmic side of the trimer center, near the $\mathrm{N}$ and $\mathrm{C}$ termini, a cluster of six phenylalanines, formed by residues 73 and 75 of each monomer, is found. Here, the aromatic rings from adjacent monomer interfaces are mutually perpendicular to each other, optimizing quadrupole interactions ${ }^{30}$ and connecting adjacent monomers.

In ScrY and all E. coli porins, loop L2 fills a gap above L3 of a neighboring monomer ${ }^{11,21}$. This loop contributes considerably to the stability of the trimer because it connects adjacent porins in a manner similar to an outward-bent hook.

\section{The membrane exposed surface}

The peripheral transmembrane surface of the ScrY porin trimer can be divided into three zones that encircle the trimer: a hydrophobic zone with a width of up to $33 \AA$ composed of aliphatic residues (such as $\mathrm{Ala}$, Leu, Ile and Val) and two hydrophilic zones at the periplasmic and external surfaces. Two aromatic girdles form borderlines between the hydrephobic zone and the two hydrophilic zones (Fig. 2a). A neutron diffraction analysi ${ }^{31}$ showed that the hydrocarbon core of detergent micelles just covers the hydrophobic zone of OmpF porin, which is thus identified as the contact interface with the lipid chains in the outer membrane. The accumulation of aromatic amino acids at the polar/apolar border is a common feature of all known structures of porins and is also seen, but is less obvious in other membrane protein ${ }^{32}$. These girdles are thought to seal the porins into the outer membrane lipid bilayer as the 'amphiphilic' aromatic residues are well suited for partitioning into the interphase between the hydrocarbon and polar membrane layers ${ }^{10,12}$. The comparison with maltoporin (Fig. $2 b$ ) and with nonspecific porins shows that the girdles in ScrY are much less regular, with more aromatic residues in the hydrophobic zone. We speculate that the irregularity of the girdles in ScrY may contribute to the specific toxicity of ScrY to E. coli cells at high expression levels ${ }^{26}$ since this would likely impair the sealing of the porins with the membrane lipid bilayer, possibly resulting in membrane instability or porin accumulation somewhere before the outer membrane.

\section{The 'greasy slide' and its 'ionizable track'}

Schirmer et al. ${ }^{21}$ identified six aromatic residues on the inner surface wall of the maltoporin from the external vestibule across the constriction site toward the periplasmic vestibule. As sugar binding by proteins often involves stacking of pyranosyl rings on aromatic rings ${ }^{33}$ these authors proposed that these aromatics are a absolutely conserved (Trp 151, Tyr 118, Tyr 78, Trp 482; Fig. 3), even up to the level of a similar side chain conformation. The fifth, $\operatorname{Trp} 358$, is conservatively replaced by Phe 435 whereas the sixth near the periplasmic mouth is missing. Following Wang et $a l .^{23}$, we use the terms S1-S5 for the glucosyl binding sites near Trp 151, Tyr 118, Tyr 78, Trp 482 and Phe 435.

The structure of the residues lining the constriction site $(78-80,110,114,118,120-121,140,142,161,187-188$, $194-201,204,207,322-323$ ) is rather similar in both glycoporins. Of these residues, all except three are conserved or conservatively replaced and most adopt a similar side chain conformation in ScrY and maltoporin. In spite of the very similar architecture of both glycoporins around the constriction site, the cross section of the channel in ScrY is larger $(8.5 \AA \times 11 \AA)$ than in maltoporin $(7 \AA \times 10 \AA)$. This is due to three major exchanges of L3-residues protruding into the lumen: Asn 192 in ScrY becomes Arg 109 in maltoporin, but with a reversed orientation so that the side chain is oriented into the lumen in maltoporin; Asp 201 and Phe 204 in ScrY are replaced by Tyr 118 and Asp 121 of maltoporin, the latter two residues protruding more into the constriction site lumen than the former. The larger cross section of the constriction site and the wider external channel mouth qualitatively explain the much higher single channel conductance of ScrY in $1 \mathrm{M} \mathrm{KCl}(1,4 \mathrm{nS})$ as compared to maltoporin $(0,15 \mathrm{nS})^{28}$.

In the neighborhood of the greasy slide, the degree of conservation of ionizable residues - the 'ionic track' of Schirmer ${ }^{23}$ is high and above the average conservation found between ScrY and maltoporin. Many of these are conserved even in their side chain conformation between ScrY and maltoporin. Some of them were found to be involved in hydrogen bonding of two maltose molecules, a maltotriose molecule, a maltohexaose molecule and a sucrose molecule bound to maltoporin ${ }^{22,23}$.

\section{Sucrose binding sites in ScrY}

The structure of ScrY cocrystallized with sucrose reveals two sucrose molecules, 'Suc1' and 'Suc2', bound to the channel surface (Fig. $4 a$ ) with occupancies of 0.7 and 0.8 respectively. Their glucosyl groups are in van der Waals contact with Tyr 78 (S3), located in the constriction site, and with $\operatorname{Trp} 482$ (S4), adjacent to the periplasmic end of the constriction site (Figs 5, 6b), the same sites as for the glucosyl groups ( $\mathrm{g} 3$ and $\mathrm{g} 4$ ) of a maltose found in maltoporin ${ }^{22}$. The sucrose molecules form hydrogen bonds with the ionizable track in the neighborhood of the greasy slide and of L3, as shown schematically in Fig. 7.

Suc1 and Suc2, as well as a single sucrose molecule in malto- 
Fig. 5 Constriction site viewed (stereo) from the periplasmic space with superimposed electron density map. Suc2 is seen in the front bound near Trp 482 ( $\$ 4$ binding site), Suc1 is seen in the background near Tyr 78 ( 53 binding site). Green : $2 \boldsymbol{F}_{\text {obs }}-\boldsymbol{F}_{\text {calc }}$ density at $2.0 \sigma$. Cyan : $\boldsymbol{F}_{\text {obs }}$ $\boldsymbol{F}_{\text {calc }}$ 'omit' density at $1.5 \sigma$ of the ScrY-sucrose complex with phases calculated after refinement of the model without sucrose.

porin ${ }^{23}$, are all similarly positioned with the glycosyl group nestled against the greasy slide, the fructosyl group bending away from it and the nonreducing glucosyl-C4 pointing towards the periplasm. The greasy slide seems to bind the sugars in a defined direction, that is with the nonreducing end towards the periplasm.

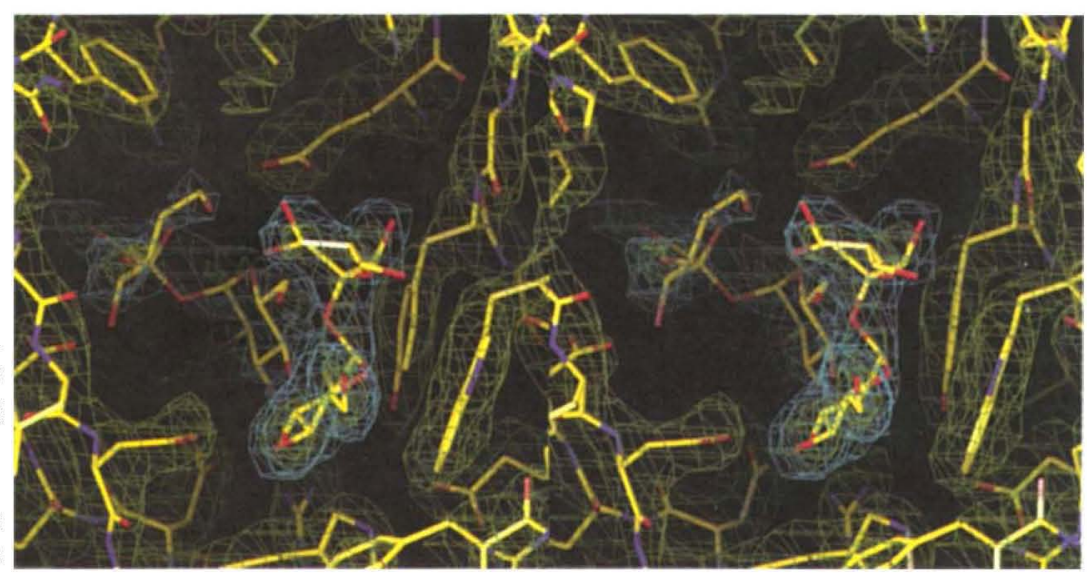
This orientation has been found for all bound sugars in glycoporins $^{22-24}$.

Sucrose binding to $\$ 3$ is sterically blocked in maltoporin due to the presence of Tyr 118 and Arg 109, both of which protrude into the lumen of the constriction site (Fig. $6 a$ ). These maltoporin residues as well as Asp 121 are represented in Fig. $6 a$ by their transparent van der Waals surface. In addition to rendering the binding of sucrose to $\$ 3$ sterically impossible, these residues of maltoporin represent a 'bolt' in the constriction site blocking the path between $\$ 3$ and S4. The remainder of this region is very similar in both glycoporins. Indeed, the glucosyl group of the single sucrose molecule found by Wang et al..$^{23}$ in maltoporin binds to a position between S2 and S3, just before the constriction site.

From the very low permeation rate of sucrose through maltoporin, one can infer that an extended conformation of sucrose, which should permeate through maltoporin efficiently, is very unfavorable. Our results demonstrate how ScrY, in contrast to maltoporin, is constructed to allow the bulky sucrose to slide easily through the channel. The observation that, in the absence of ScrY, E. coli cannot grow on low sucrose concentrations as sole carbon source ${ }^{26}$ shows that ScrY achieves a high permeability for this disaccharide.

\section{Other protein-sucrose complexes}

In contrast to maltose and maltoligosaccharides, sucrose in crystalline form and bound to proteins has a globular conformation with the glucosyl and the fructosyl moieties bent towards each other. The torsion angles, $\Phi, \Psi$, of sucrose ${ }^{34}$ are found to be similar in crystallographical and molecular modelling studies to the values of crystalline sucrose $\left(108^{\circ} /-45^{\circ}\right)$, indicating a similar overall conformation in solution and in the crystal ${ }^{34-36}$. In ScrY, the torsion angles about the two glycosidic bonds of sucrose, $\Phi$, $\Psi$, are found to be $75^{\circ} /-31^{\circ}$ for Suc1 and $90^{\circ} /-61^{\circ}$ for Suc2.

In a complex with lentil-lectin ${ }^{37}$, sucrose exhibits torsion angles of $107^{\circ}$ and $-58^{\circ}$, with a water intercalated between glucosyl-O2 and fructosyl-O3. All of the glucosyl hydroxyls are hydrogen bonded to the protein but only two fructose hydroxyls form indirect hydrogen bonds to the protein through waters. Moreover, the glucosyl group is stacked on a phenylalanine ring. The features of the bound sucrose in lentil-lectin are also found in ScrY and maltoporin (Fig. 7). The two glucosyl groups are more intimately attached to the channel wall than the fructosyl groups, that is they are in van der Waals contact with aromatic residues and form a larger number of hydrogen bonds to the protein. As the fructosyl group contributes less to the free energy of

binding and since maltose can be considered as two glucosyl groups the binding of maltose to ScrY should be stronger than that of sucrose to ScrY. Indeed, experimental determination of the respective binding constants ${ }^{28}$ yield $K_{\mathrm{D}}$ values of $6.25 \mathrm{mM}$ and $50 \mathrm{mM}$ for maltose and sucrose.

Oligosaccharide binding sites involving chains of aromatic residues flanked by potential hydrogen bond forming residues have also been found in other proteins. Cellulose-binding domains of cellulolytic enzyme complexes from fungi and bacteria indicated binding sites for three adjacent crystalline cellulose chains with aromatic residues interacting with roughly every second glucose unit of one chain ${ }^{38}$. E. coli maltodextrin phosphorylase shows stacking of a glucosyl group on a $\mathrm{Tyr}^{39}$. The authors discuss two factors contributing to the favorable stacking of glucosyl groups on aromatic residues: hydrophobic interactions and electrostatic interactions between partial positive charges on the hydrogens bound to carbons of the sugar ring and the $\pi$ electrons of the aromatic ring.

On the other hand, chains of aromatic residues are not a general feature of oligosaccharide binding, as seen from the structure of a bacterial endoglucanase ${ }^{40}$, and lysozyme ${ }^{41}$. C-type ( $\mathrm{Ca}^{2+}$-dependent) lectins show how the surroundings of a $\mathrm{Ca}^{2+}$ atom, bound to the surface of a protein, can create a low-affinity binding site for monosaccharides ( $K_{\mathrm{D}}$ between 6 and $22 \mathrm{mM}$ ) which can bind two neighboring equatorial sugar hydroxyls to the protein ${ }^{42,43} \cdot \mathrm{Ca}^{2+}$ has also been found to be involved in indirect binding of glucose through water molecules ${ }^{44}$ or by apparently optimizing the conformation of residues for hydrogen bonding to sugar hydroxyls ${ }^{45}$.

Glycoporins possess a series of low-affinity binding sites for glucosyl groups. Binding occurs through hydrophobic contacts with aromatic residues and hydrogen bonding to ionizable residues. The binding sites are arranged as an extended slide from the extracellular mouth to the periplasmic mouth of the channel. The observed increase of binding constants for maltooligosaccharides with increasing number of glucosyl residues in maltoporin ${ }^{20}$ and $\mathrm{ScrY}^{28}$ suggests a series of four or five binding sites with the correct spacing and geometry to bind such helical maltooligosaccharide chains ${ }^{21,24}$. As binding sites increase the sugar permeability of the outer membrane, it is expected that glycoporins confer a growth advantage to bacteria whose growth is limited by low concentration in the culture medium of disaccharides like maltose or sucrose or maltotriose and may be indispensable for the uptake of maltooligosaccharides with more than three glucosyl residues. Indeed in the presence of maltooligosaccharides longer than three glucose units as the only available car- 

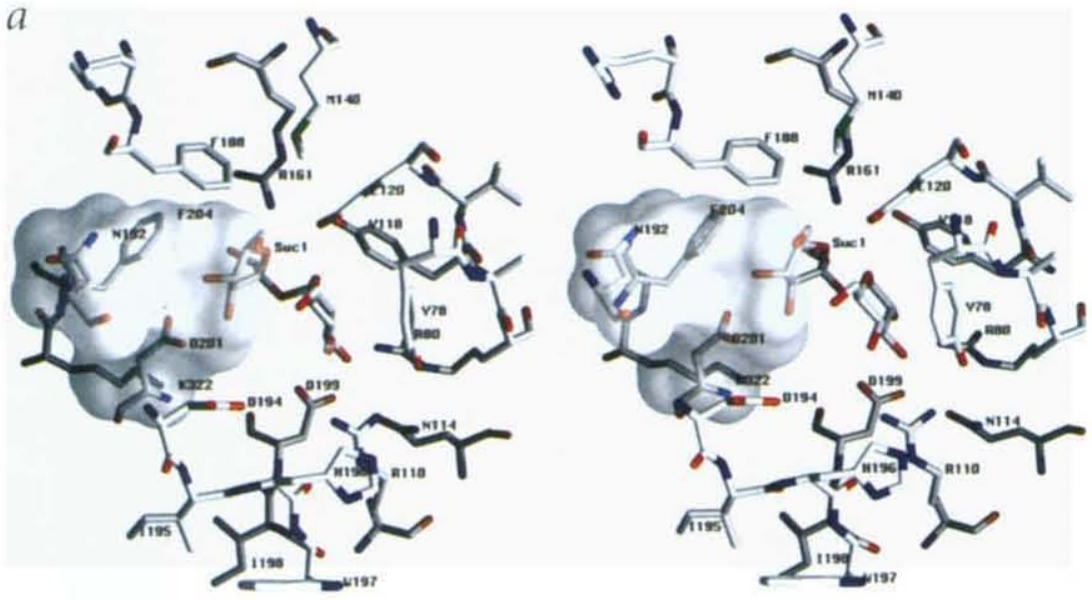

$b$

si

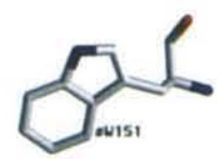

$\mathbf{s 2}$

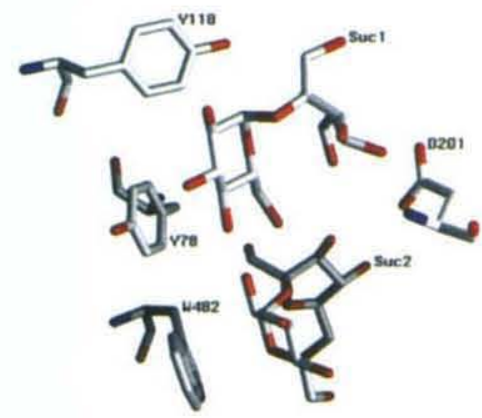

Ss

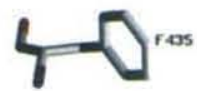

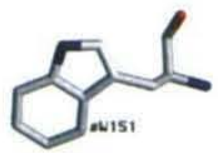
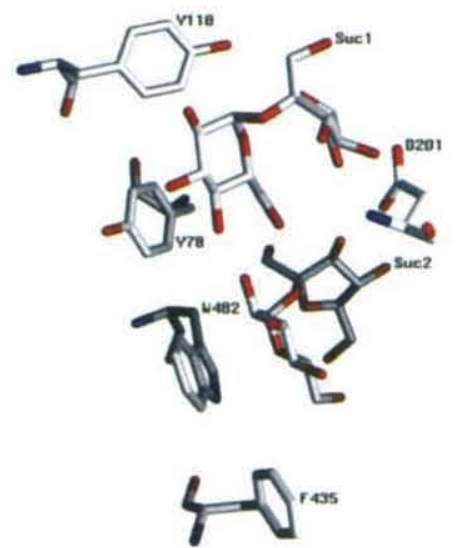

Fig. 6 a, Constriction site viewed (stereo) from the periplasmic space with Suc1 bound to S3. The residues forming the constriction site are shown. All of these, except for Asn 192, Asp 201 and Phe 204, are conserved in maltoporin up to a similar side chain conformation. The space occupied by the three altered residues (Arg 109, Tyr 118, Asp 121 in maltoporin) is indicated by its transparent van der Waals surface. $\boldsymbol{b}$, Greasy slide, stereo side view with the external side oriented to the top, as represented by aromatic residues Trp 51, Tyr 118, Tyr 78, Trp 482, Phe 435 (S1-S5) with Suc1 bound near Tyr 78 (S3) and Suc2 bound near Trp 482 (S4). This figure was prepared with GRASP78.

and complexed structures can be overlayed with an r.m.s.d. of $0.24 \AA$, which is the same value as found when comparing all side chains. This rigidity of the residues lining the sucrose path should minimize frictional forces on the sliding sugar molecule by the protein. According to the reaction rate theory of Kramers, a low frictional coefficient in an overdamped protein system ensures a high reaction rate ${ }^{16}$ that results in a high channel permeability.

\section{Division of permeation into four reactions}

A full description of permeation should include the following consecutive reactions :

(i) Diffusion of sugars from the external medium to the vestibule.

(ii) Collisions with and diffusion on the vestibule surface resulting in binding to the external end of the greasy slide.

(iii) One-dimensional diffusion along the greasy slide.

(iv) Escape of sugar from the periplasmic end of the greasy slide.

bon source, $E$. coli cells need maltoporin or ScrY for growth ${ }^{26}$. It is comprehensible to ascribe this requirement to the greasy slide, as permeation of these longer chain molecules with their high entropy of threading through the channel will depend upon a high-affinity binding site. As Schirmer et al. have pointed out, the complementarity of the greasy slide geometry to the natural left-handed superhelical conformation of maltooligosaccharides is one feature underlying the high affinity ${ }^{21}$.

The binding sites with the highest affinities are found close to the constriction site. This is in accord with the high entropy barrier associated with threading the sugar molecule through this zone. Binding at any position on the greasy slide may be 'flexible'; that is, sugars might find alternative hydrogen bonding partners at similar free energies. Mutational studies are in accord with this view, as point mutations of aromatic residues of the 'greasy slide' in maltoporin do not inhibit uptake of maltooligosaccharides ${ }^{46,47}$. The resulting free-energy profile of a glucosyl unit sliding along the chain of binding sites seems to be smooth, with only shallow barriers in between sites ${ }^{48}$.

Interestingly, differences in side chain conformations between residues of the native and sucrose-complexed form are only slightly greater than those of residues not involved in sucrose binding. The side chain conformations of sucrose-binding residues in the native
At equilibrium the flux of sugar through each partial reaction is equal and expected to result from evolutionary optimization for high permeability. The apparently low frictional coefficient of the sliding reaction (iii) could be a consequence of such an optimization.

The larger the concentration gradient driving the first reaction, the better is the process optimized. It seems not to be perfect yet as Jordy et al. ${ }^{49}$ have concluded from the order of magnitude of the on-rate constants for maltooligosaccharide binding that the sugar transport is not limited by the diffusion to the channel entrance; in other words, that the sugar hits the channel many times before it is bound to the greasy slide in the constriction site. Nevertheless the external vestibule should be engineered to minimize the number of hits of sugar molecules needed for a successful capturing. If maltoporin and ScrY have been optimized for capturing different sugars, their respective vestibule surfaces should differ in their respective coating with residues. Maltooligosaccharides that represent an extended ribbon-like structure with one face being less polar might for instance stick to patches of aromatic residues. Sucrose molecules with their globular shape and their numerous hydrogen bonding groups might preferentially stick to ionizable residues. A weak association of the sugar with the vestibular surface could 


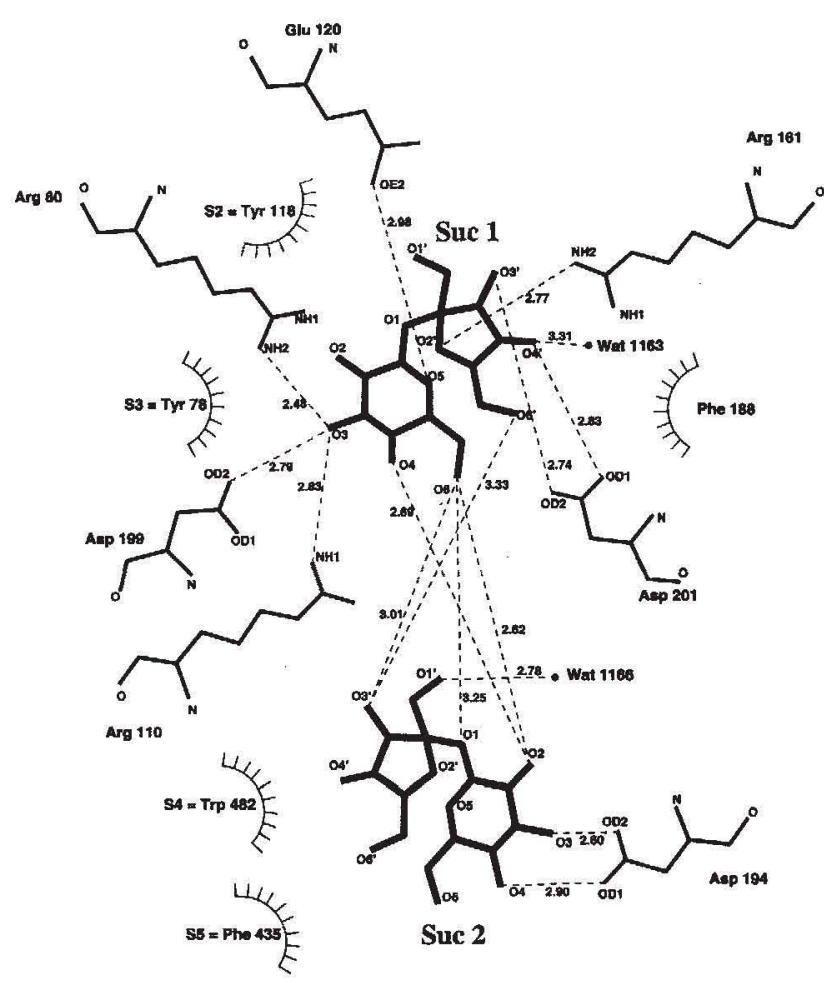

Fig. 7 LIGPLOT $^{9}$-style scheme of hydrogen bonding network of Suc1 bound to 53 and SuC2 bound to $\$ 4$.

improve the diffusional flow of sugars through reaction (ii) ${ }^{50}$. In Fig. 4 both glycoporins are shown with aromatic residues at the surface coded in green and ionizable residues (Asp, Glu, Arg, Lys) coded in red. Indeed, maltoporin has a noticeable density of aromatic residues on its vestibule surface (see also Fig. 2) while in $\mathrm{ScrY}$ ionizable residues predominate.

\section{The periplasmic side of ScrY}

Schülein et al. ${ }^{28}$ constructed a deletion mutant ScrY $\Delta 3-73$ and studied its properties. The deletion increased the cation versus anion permeability $\mathrm{P}_{\mathrm{c}} / \mathrm{P}_{\mathrm{a}}$ and increased the association with the peptidoglycan.

Due to absence of electron density, the N-terminal 70 residues of the ScrY crystal structure could not be traced and modeled. Inspection of the sequence shows a segment with a clear heptad ' $a$ '-' $g$ ' pattern (A. Lupas, personal communication) indicating a $99 \%$ probability for a coiled-coil tertiary structure ${ }^{51}$ for 42 residues starting at Ile 4 . The heptads are indicated in the diagram in Fig. 8. Hydrophobic residues Leu, Ile, Ala, Val are on positions ' $a$ ' and ' $d$ ', while Glu residues are on position ' $e$ ' and Arg and one Lys on position ' $g$ '. No structural prediction exists for the segment between Gln 46 and Lys 70.

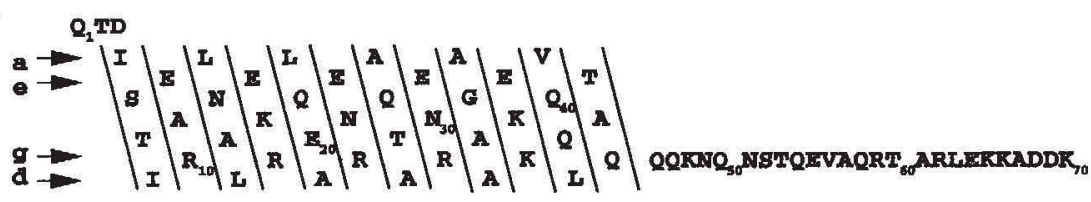

Fig. 8 Sequence of the N-terminal 70 residues of ScrY showing heptad pattern of residues 4-45.
It appears likely that three $\alpha$-helices, one from each $S_{\mathrm{cr} Y}$ monomer, associate to form a left-handed coiled-coil. The latter would consist of $12 \alpha$-helical turns and have a length of roughly $65 \AA$. Assuming an interhelical distance of $11 \AA$ (ref. 52) and a diameter of $12 \AA$, the cross section of the coiled-coil would be roughly $23 \AA$. The packing of the protein in the unit cell allows accomodation of such a cylindrical volume without steric problems.

The length of the $\mathrm{N}$-terminal extension is much shorter than the $400 \AA$ predicted for the coiled coil of the periplasmic lipoprotein Omp $\alpha$ from Thermotoga maritima ${ }^{53}$, shorter than the estimated distance of $200 \AA$ (ref. 53) between the outer and inner membrane of $E$. coli, shorter than the coiled-coil predicted within the II-domain of TolA which was reported to bind porins $s^{54}$ and of roughly the same length as the coiled-coil predicted for the murein lipoprotein (Braun's lipoprotein) of E. coli ${ }^{55,56}$.

ScrY is coexpressed with a sucrose specific PTS system for which no periplasmic binding protein is known. Free diffusion of the sugars in the periplasmic space would reduce the efficiency of sugar uptake as some sugar molecules would diffuse back to the external phase. We speculate that the N-terminal coiledcoil functions as a low affinity binding site for sugars, effectively replacing a sucrose-specific periplasmic binding protein. The oppositely ionizable residues on the ' $e$ ' and ' $g$ ' heptad positions of the coiled-coil are potential hydrogen bonding partners for sugar hydroxyls. Oppositely ionizable residues on ' $e$ ' and ' $g$ ' positions have been shown to contribute to the stability of other coiled-coils ${ }^{57}$ in accord with crystal structures where these charged pairs form salt bridges ${ }^{58}$. However, theoretical considerations $^{59}$ and $\mathrm{pK} K_{\mathrm{a}}$ shift values of acidic residues in coiled-coils ${ }^{60}$ suggest that these ionizable residues remain solvated and thus are potential hydrogen bonding partners for sugar hydroxyls in vivo. The question if they really form low-affinity sugar binding sites must be answered experimentally.

\section{Methods}

Crystallization and data collection. The isolation and purification of ScrY is as previously described ${ }^{29,61}$. ScrY was crystallized by vapor diffusion using the sitting drop method at $17{ }^{\circ} \mathrm{C}$. The drop contained 5-7 mg ml-1 protein, $20 \mathrm{mM}$ Tris $/ \mathrm{Cl}$ at pH 7.7, $100 \mathrm{mM} \mathrm{LiCl,} 20 \mathrm{mM}$ $\mathrm{MgSO}_{4}, 1.2 \% \quad \beta$-D-octylglucopyranoside ( $\beta$-OG), at least $1 \%$ Hexyldimethylamineoxide ( $\left.C_{6} \mathrm{DAO}\right), 1 \% \beta$-D-heptylglucopyranoside $(\beta-H G)$ and $6-9 \%$ PEG-2000. The concentration of PEG in the reservoir was $12-15 \%$. For cocrystallization with sucrose, we added $100 \mathrm{mM}$ sucrose to the drop. Heavy atom derivatives were produced by soaking crystals or by cocrystallizing the protein in the presence of the heavy metal compound. Crystals grew within three to six weeks to a size up to $400 \mu \mathrm{m}$ in each direction. The space group is $\mathrm{P}_{2}(a=b=$ $112.1 \pm 1.5 \AA, c=147.0 \pm 2 \AA$ ) with one trimer per asymmetric unit. The Matthews-parameter of the crystal has a value of $3.8 \AA^{3} / \mathrm{Da}$, indicating a solvent (plus detergent) content of $68 \%$.

The crystals diffracted to better than $2.0 \AA$ resolution using synchrotron radiation (EMBL beamline X11 at DESY, Hamburg, Germany). The native data for the initial structure solution reported here as well as the heavy atom derivatives and sucrose data were collected on an image-plate-detector system (STOE, Darmstadt, Germany) using CuKa-radiation from a rotating anode generator (40 $\mathrm{kV}, 100 \mathrm{~mA}$ ). Crystals were aligned with the $c$ axis along the spindle, and frames of $0.5^{\circ}$ oscillation were collected over $65^{\circ}$ at $14^{\circ} \mathrm{C}$. The resulting images were processed with XDS62.

Crystallographic procedures. Heavy atom binding sites were located in difference Patterson maps and refined using DAREF ${ }^{63}$. The multiple isomorphous replacement (MIR) phases were improved by solvent flattening 


\begin{tabular}{|c|c|c|c|c|c|c|c|c|c|}
\hline \multicolumn{10}{|c|}{ Table 1 Crystallographic data } \\
\hline \multirow[t]{2}{*}{ Dataset } & \multirow[t]{2}{*}{$\begin{array}{l}\text { Twinning } \\
\text { fraction (\%) }\end{array}$} & \multirow[t]{2}{*}{ Resolution $(\AA)$} & \multirow[t]{2}{*}{$\begin{array}{c}\text { Completeness } \\
(\%)\end{array}$} & \multirow[t]{2}{*}{$\begin{array}{l}\text { Number of } \\
\text { reflections }\end{array}$} & \multirow[t]{2}{*}{$\begin{array}{c}\text { Number of } \\
\text { sites }\end{array}$} & \multirow[t]{2}{*}{$\begin{array}{l}R_{\text {sym }}{ }^{1} \\
(\%)\end{array}$} & \multirow[t]{2}{*}{$\begin{array}{l}R_{\text {iso }}{ }^{2} \\
(\%)\end{array}$} & \multicolumn{2}{|c|}{$\begin{array}{l}\text { Phasing power } \\
\text { in shells }\end{array}$} \\
\hline & & & & & & & & $99-4 \AA$ & $4-2.9 \AA$ \\
\hline Native 0 & 0 & 2.89 & 83.5 & 38,033 & - & 10.4 & - & - & - \\
\hline Native 1 & 3 & 2.89 & 93.5 & 42,612 & - & 9.7 & 29.9 & - & - \\
\hline Native 2 & 3 & 2.29 & 92.6 & 82,849 & - & 8.3 & 24.9 & - & - \\
\hline Sucrose soak & 3 & 2.29 & 78.7 & 72,892 & - & 16.1 & 30.1 & - & - \\
\hline $\mathrm{KAu}(\mathrm{CN})_{2}$ & 3 & 2.89 & 83.7 & 38,145 & 9 & 9.7 & 28.0 & 2.2 & 1.3 \\
\hline cis- $\mathrm{Pt}\left(\mathrm{NH}_{3}\right)_{2} \mathrm{Cl}_{2}$ & 3 & 2.89 & 95.7 & 43,612 & 9 & 11.3 & 29.1 & 1.2 & 0.7 \\
\hline $\mathrm{K}_{2} \operatorname{ReCl}_{4}$ & 12 & 2.89 & 85.4 & 38,912 & 9 & 10.6 & 23.4 & 1.1 & 0.6 \\
\hline
\end{tabular}

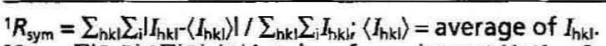

${ }^{2} R_{\mathrm{sis}}=\left.\sum|I| I_{\mathrm{p}}\left|/ \sum\right| I_{\mathrm{p}}\right|_{i}\left|I_{\mathrm{p}}\right|$ is taken from dataset Native 0 .

3Phasing power is mean value of heavy atom structure factor amplitude divided by lack of closure.

${ }^{4}$ Reference data to determine the twinning fraction $\alpha$ and $R_{\text {iso }}$ was data set Native 0 .

and non-crystallographical symmetry (NCS) averaging, using RAVE ${ }^{64}$ or $\mathrm{DM}^{65,66}$. Skeletonizing and model building were done with the program $O^{67}$. The model was refined using $X-P L O R^{68}$ using published protocols ${ }^{69}$. Water molecules were identified in difference density maps and only included into the model if they were present in all three monomers. $2 \boldsymbol{F}_{\text {obs }}-\boldsymbol{F}_{\text {cak }}$ electron density maps were averaged over the three monomers of the asymmetric unit. The final model of uncomplexed ScrY was used for finding sucrose binding sites in datasets of sucrose-complexed ScrY. Anisomorphism between uncomplexed and sucrose-complexed crystals was dealt with by performing 20 cycles of rigid-body minimization prior to electron density map calculation. $70 \mathrm{~N}$-terminal residues in each monomer were not included into the model, as there was no interpretable electron density for this segment. Solvent molecules and one $\mathrm{Ca}^{2+}$ atom per monomer were added in the final stages of refinement. All atoms were under strong NCS-restraints during the refinement.

Analysis of twinning. In $\mathrm{P}_{(1,2)}$, four non-equivalent ways to index a given reflection exist, described by $(\mathrm{h} \mathrm{kl})(\mathrm{k} h-1)(-\mathrm{h}-$ $k 1)$ or $(-k-h-1)$. For each dataset, only one of these possibilities is suitable for merging with other datasets or difference coefficient calculation, and can be identified on the basis of a low $R$-factor with respect to a reference dataset. For some of these calculations we found that not just one of these possibilities yielded a low $R$-factor but a second also gave an $R$-factor better than that expected for unrelated datasets. The second possibility always corresponded to a rotation of the unit cell by $180^{\circ}$ about the axis bisecting the unit cell vectors $a$ and $b$ : $(h \rightarrow k$, $k \rightarrow h, \mid \rightarrow-1)$. Thus, a hemihedral twinning law of the form

$I_{\text {obs }}(h|k|)=(1-\alpha) I_{\text {true }}(h k l)+\alpha I_{\text {true }}(k h-l)$

with a twinning fraction $\alpha$ has to be used to describe the intensities of ScrY datasets. Detwinning can be done using

$I_{\text {true }}(h k I)=\left((1-\alpha) I_{\text {obs }}(h k I)+\alpha I_{\text {obs }}(k h-I)\right) / 1-2 \alpha$

as long as the twinning fraction a is markedly lower than 0.5 . Determination of $\alpha$ by intensity statistics ${ }^{70}$ was not possible as the non-crystallographic symmetry axis nearly coincides with the twinning operation. Therefore $\alpha$ was determined with a method similar to that of Gomis-Rüth et al..$^{71}$ as follows:

(i) For all native datasets, the correlation coefficient between the $(\mathrm{h} \mathrm{k})$ and the $(\mathrm{k} \mathrm{h}-\mathrm{l})$ reflections (or their symmetry mates) was evaluated; and the dataset with the lowest correlation was defined as the reference. Intensities $I_{\text {ref }}$ from this dataset were preliminarily assumed to be unaffected by twinning.

(ii) For each measured dataset with intensities $I^{\prime}$, the $R$-factor

$$
R(\alpha)=\left(\Sigma\left|I^{\prime}-I(\alpha)\right|\right) / \Sigma I^{\prime}
$$

with $\mathrm{I}(\alpha)=(1-\alpha) \mathrm{I}_{\text {ref }}(\mathrm{h} \mathrm{k} \mathrm{I})+\alpha \mathrm{I}_{\text {ref }}(\mathrm{k} h-\mathrm{l})$ with respect to the reference dataset with intensities $I_{\text {ref }}$ was calculated, and the value of $\alpha$ that gave the lowest $R(\alpha)$ was taken as the twinning fraction of this dataset. The accuracy of $\alpha$ determined by this procedure was checked with model calculations, and found to be correct within a few percent over the range $\alpha=0-0.5$. Furthermore, the procedure gave good results even in the case of anisomorphism or heavy atom substitution. As an alternative to $\mathrm{R}(\alpha)$, we also used the correlation coefficient between I' and I $(\alpha)$, with similar results.

(iii) When a model for ScrY porin became available, the $\boldsymbol{F}_{\text {calc }}$ derived from the model were used as the $\alpha=0$ reference. In this way, the twinning fraction of the previously used reference dataset was found to be $\alpha=1 \%$.

Furthermore, the final model of ScrY was subjected to SHELXL-

Table 2 Crystallographic refinement statistics

\begin{tabular}{|c|c|c|}
\hline & native & sucrose-bound \\
\hline Resolution ( $\AA$ ) & $15-2.4$ & $15-2.4$ \\
\hline $\mathrm{P}_{2}$ cell axes $(\AA)$ & $112.1,112.1,147.0$ & $111.8,111.8,147.8$ \\
\hline Twinning fraction ${ }^{1} \alpha$ & 0.011 & 0.018 \\
\hline$R$-factors $R_{\text {free }} / R_{\text {cryst }}(\%)$ & $22.8 / 21.4$ & $24.6 / 20.7$ \\
\hline Number of reflections $(F>\sigma F)$ for $R_{\text {free }} R_{\text {cryst }}$ & $1,036 / 62,256$ & $891 / 54,444$ \\
\hline Completeness overall $(\boldsymbol{F}>\sigma \boldsymbol{F})(\%)$ & 78.3 & 67.4 \\
\hline \multicolumn{3}{|l|}{ Number of: } \\
\hline Non-hydrogen protein atoms & 9,606 & 9,606 \\
\hline Sucrose atoms & 0 & 138 \\
\hline Solvent molecules & 330 & 330 \\
\hline Calcium ions & 3 & 3 \\
\hline \multicolumn{3}{|l|}{ R.m.s. deviation from ideality: } \\
\hline Bond length $(\AA)$ & 0.010 & 0.011 \\
\hline Bond angles $\left(^{\circ}\right)$ & 1.68 & 1.73 \\
\hline Dihedral angles $\left({ }^{\circ}\right)$ & 28.3 & 28.4 \\
\hline $\begin{array}{l}\text { R.m.s. deviation of } B \text {-factors of covalently } \\
\text { bound atoms }\left(\AA^{2}\right)\end{array}$ & 1.8 & 1.9 \\
\hline $\begin{array}{l}\text { R.m.s. deviation from non-crystallographic } \\
\text { symmetry, all atoms }(\AA)\end{array}$ & 0.10 & 0.10 \\
\hline
\end{tabular}

1Resulting from SHELXL-96 refinement of $\alpha$

${ }^{2} R_{\text {cryst }}=\Sigma\left\|\boldsymbol{F}_{\mathrm{o}}|-| \boldsymbol{F}_{\mathrm{c}}\right\| / \sum\left|\boldsymbol{F}_{\mathrm{o}}\right|$ where $\left|\boldsymbol{F}_{\mathrm{o}}\right|$ and $\left|\boldsymbol{F}_{\mathrm{c}}\right|$ are the observed and scaled calculated structure factor amplitude respectively. 
9672 refinement, with the twinning fraction $\alpha$ used as a refineable parameter in the program, thus treating twinning in a mathematically adequate way.

Coordinates. The coordinates of the complexed and uncomplexed forms have been deposited in the Brookhaven Protein Data Bank (accession codes 1AOT and 1AOS respectively.)

1. Lugtenberg, B. \& Van Alphen, L. Molecular architecture and functioning of the outer membrane of Escherichia coli and other gram-negative bacteria. Biochim. Biophys. Acta 737, 51-115 (1983).

2. Bayer, M.E. \& Bayer, M.H. in Bacterial Cell Wall (eds Ghuysen, J.-M. \& Hakenbeck, R.) 447-462 (Elsevier Sci. B.V., Amsterdam; 1994).

3. Riley, M. \& Labedan, B. in Escherichia coli and Salmonella (ed. Neidhardt, F.C) 2118-2202 (ASM Press, Washington, D.C.; 1996).

4. Boos, W. \& Lucht, J.M. in Escherichia coll and Salmonella (ed. Neidhardt, F.C.) 1175-1209 (ASM Press, Washington, D.C.; 1996).

5. Postma, P.W., Lengeler, J. \& Jacobson, G.R. in Escherichia coli and Salmonella (ed. Neidhardt, F.C.) 1149-1174 (ASM Press, Washington, D.C.; 1996)

6. Benz, R. \& Bauer, K. Permeation of hydrophilic molecules through the outer membrane of gram-negative bacteria. Eur...Biochem. 176, 1-19 (1988).

7. Welte, W., Nestel, U., Wacker, T. \& Diederichs, K. Structure and function of the porin channel. Kidney Int. 48, 930-940 (1995).

8. Delcour, A.H., Adler, J., Kung, C. \& Martinac, B. Membrane-derived oligosaccha rides (MDO's) promote closing of an E. coli porin channel. FEBS Lett. 304, 216-220 (1992)

9. Welte, W., Diederichs, K., Przybylski, M., Glocker, M., Benz, R. \& Breed, J. X-ray crystallographic and mass spectrometric structure determination and function characterization of succinylated porin from $R$. capsulatus : Implications for ion selectivity and single-channel conductance. In Proceedings of the NATO Advanced workshop "New Methods for the Study of Molecular Aggregates" (eds Standing, K. \& Ens, W.) in the press.

10. Weiss, M.S. et al. Molecular architecture and electrostatic properties of a bacterial porin. Science 254, 1627-1630 (1991)

11. Cowan, S. W. et al. Crystal structures explain functional properties of two E. coli porins. Nature 358, 727-733 (1992)

12. Kreusch, A., Neubüser, A., Schiltz, E., Weckesser, J. \& Schulz, G. E. Structure of the membrane channel porin from Rhodopseudomonas blastica at $2.0 \AA$ resolution. Protein Sci. 3, 58-63 (1994).

13. Hirsch, A., Diederichs, K., Breed, J., Saxena, K., Richter, O.-M. Ludwig, B. \& Welte $W$. The structure of porin from Paracoccus denitrificans at $3.1 \AA$ resolution. FEBS Lett. 404, 208-210 (1997).

14. Eisenberg, G. \& Dani, J.A. An Introduction to Molecular Architecture and Permeability of Ion Channels. Ann. Rev. Biophys. Biophys. Chem.16, 205-226 (1987)

15. Glasstone, 5., Laidler, K.J. \& Eyring, H. The Theory of Rate Processes (McGraw Hil Book Comp., New York and London; 1941)

16. Frauenfelder, H. \& Wolynes, P.G. Rate Theories and Puzzles of Hemeprotein Kinetics. Science 229, 337-345 (1985).

17. Rosenbusch, J. P. Characterization of the major envelope protein from Escherichia coli. J. Biol. Chem. 249, 8019-8029 (1974).

18. Birge, E. Bacterial and Bacteriophage Genetics (Springer Verlag Berlin; 1981).

19. Death, A., Notley, L. \& Ferenci, T. Derepression of LamB protein facilitates oute membrane permeation of carbohydrates into Escherichia coli under conditions of nutrient stress. J. Bacteriol. 175, 1475-1483 (1993).

20. Benz, R, Schmid, A. \& Vos-Scheperkeuter, G. H.. Mechanism of sugar transport through the sugar-specific LamB channel of Escherichia coli outer membrane. Membrane Biol. 100, 21-29 (1987).

21. Schirmer, T., Keller, T. A., Wang, Y. \& Rosenbusch, J. P. Structural basis for suga translocation through maltoporin channels at $3.1 \AA$ resolution. Science 267 512-514 (1995)

22. Dutzler, R., Wang, Y-F., Rizkallah, P. J., Rosenbusch, J. P. \& Schirmer, T. Crystal structures of the various maltooligosaccharides bound to maltoporin reveal specific sugar translocation pathway. Structure 4, 127-134 (1996).

23. Wang, Y.F., Dutzler, R., Rizkallah, P.J., Rosenbusch, J.P. \& Schirmer, T. Channel specificity: Structural basis for sugar discrimination and differential flux rates in maltoporin. J. Mol. Biol. 272, 56-63 (1997).

24. Meyer, J.E., Hofnung, M. \& Schulz G.E. Structure of maltoporin from Salmonella typhimurium ligated with a nitrophenyl-maltotrioside. J. Mol. Biol. 266, 761-75 (1997).

25. Schmid, K., Schupfner, M. \& Schmitt, R. Plasmid-mediated uptake and metabolism of sucrose by Escherichia coli K-12 J. Bacteriol. 151, 68-76 (1982)

26. Schmid, K., Ebner, R., Jahreis, K., Lengeler, J. W. \& Titgemeyer, F. A sugar-specific porin, ScrY, is involved in sucrose uptake in enteric bacteria. Mol. Microbiol. 5 . 941-950 (1991)

27. Hardesty, C. Ferran, C. \& DiRienzo, J. M. Plasmid-mediated sucrose metabolism in Escherichia coli: Characterization of scrY, the structural gene for a phosphoenopyruvate-dependent sucrose phosphotransferase system outer membrane porin. J. Bacteriol. 173, 449-456 (1991).

28. Schülein, K., Andersen C. \& Benz, R. The deletion of 70 amino acids near the $\mathrm{N}$ terminal end of the sucrose-specific porin ScrY causes its functional similarity to LamB in vivo and in vitro. Mol. Microbiol. 17, 757-767 (1995).

29. Forst, D. et al. Crystallization and preliminary X-ray diffraction analysis of ScrY, a specific bacterial outer membrane porin. J. Mol. Biol. 229, 258-262 (1993).

30. Burley, S.K. \& Petsko, G.A. Electrostatic interactions in aromatic oligopeptide contribute to protein stability. TIBTECH 7, 354-359 (1989).

31. Pebay-Peyroula, E., Garavito, R.M., Rosenbusch, J.P., Zulauf, M. \& Timmins, P.A. Detergent structure in tetragonal crystals of OmpF porin. Structure 3, 1051-1059 (1995).

\section{Acknowledgments}

We gratefully acknowledge the help by W. Kreutz, R. Benz, K. Schmid, K. Schülein, C. Andersen, C. Ulmke, C. Cohen-Addad and P. Timmins, and we thank J. Breed for critically reading the manuscript. This work was supported by a grant of the Deutsche Forschungsgemeinschaft.

32. Schiffer, M., Chang, C. H.\& Stevens, F. J. Transport proteins in bacteria: Common themes in their design. Science 258, 936-942 (1992)

33. Vyas, N.K. Atomic features of protein-carbohydrate interactions. Curr. Op. Struct Biol. 1, 732-740 (1991)

34. Engelsen, S.B., du Penhoat, C.H. \& Perez, S. Molecular Relaxation of Sucrose in aqueous Solution. J. Phys. Chem. 99, 13334-13351 (1995).

35. Brown, G.M. \& Levy, H.A. Further Refinement of the Structure of Sucrose based on Neutron-Diffraction Data. Acta Crystallogr. B29, 790-797 (1973).

36. Immel, S. \& Lichtenthaler, F.W. The Conformation of Sucrose in water: A Molecular Dynamics Approach. Liebigs Ann. 1995, 1925-1937 (1995).

37. Casset, F, et al. NMR, Molecular Modelling, and Crystallographic Studies of Lenti Lectin-Sucrose Interaction. J. Biol. Chem. 270, 25619-25628 (1995).

38. Tormo, J. et al. Crystal structure of a bacterial family-IIl cellulose-binding domain: a generai mechanism for attachment to cellulose. EMBO J. 15, 5739-5751 (1996).

39. O'Reilly, M., Watson, K.A., Schinzel, R., Palm, D. \& Johnson, L.N. Oligosaccharide substrate binding in Escherichia coli maltodextrin phosphorylase. Nature Struct. Biol. 4, 405-412 (1997)

40. Davies, G.J. et al. Structure Determination and Refinement of the Humicola insolens Endoglucanase V at $1.5 \AA$ Resolution. Acta Crystallogr. D52, 7-17 (1996).

41. Ford, L.O., Johnson, L.N., Machin, P.A., Philips, D.C. \& Tijan, R. Crystal Structure of a Lysozyme-Tetrasaccharide Lactone Complex. J. Mol. Biol. 88, 349-371 (1974).

42. Ng, K.K.-S., Drickamer, K. \& Weis, W.I. Structural Analysis of Monosaccharide Recognition by Rat Liver Mannose-binding Protein. J. Biol. Chem. 271, 663-674 (1996).

43. Weis, W.1., Drickamer, K. \& Hendrickson, W.A. Stucture of a C-type mannosebinding protein complexed with an oligosaccharide. Nature 360, 127-134 (1992)

44. Qian, M., Haser, R. \& Payan, F. Carbohydrate binding sites in a pancreatic o amylase-substrate complex, derived from $X$-ray structure analysis at $2.1 \mathrm{~A}$ resolution. Protein Sci. 4, 747-755 (1995)

45. Shaanan, B. Lis, H. \& Sharon, N. Structure of a Legume Lectin with an Ordered Nlinked Carbohydrate in Complex with Lactose. Science 254, 862-866 (1991).

46. Francis, G., Brennan, L., Stretton, S. \& Ferenci, T. Genetic mapping of starch-and lambda-receptor sites in maltoporin: identification of substitutions causing direct and indirect effects on binding sites by cysteine mutagenesis. Mol. Microbiol. 5, 2293-2301 (1991)

47. Benz, R., Francis, G., Nakae, T. \& Ferenci, T. Investigation of the selectivity of maltoporin channels using LamB proteins: Mutations changing the maltodextrin binding site. Biochim. Biophys. Acta 1104, 299-307 (1992)

48. Meyer, J.E.W. \& Schulz, G.E. Energy profile of maltooligosaccharide permeation through maltoporin as derived from the structure and from a statistical analysis of saccharide-protein interactions. Protein Sci, 6, 1084-1091 (1997).

49. Jordy, M., Andersen, C., Schülein, K., Ferenci, T. \& Benz, R. Rate Constants of Sugar Transport Through Two LamB Mutants of Escherichia coli : Comparison with Wild-type Maltoporin and LamB of Salmonella typhimurium. J. Mol, Biol. 259, 666-678 (1996)

50. Adam, G. \& Delbrück, M. : Reduction of Dimensionality in Biological Diffusion Processes. in : Structural Chemistry \& Molecular Biology 198-215 (eds Rich, A. \& Davidson, N.) (Freeman, San Francisco; 1968).

51. Lupas, A. Coiled coils: New structures and new functions. TIBS 21, 375-382 (1996).

52. DeLano, W. L. \& Brünger A. T. Helix packing in Proteins: prediction and energetic analysis of dimeric, trimeric, and tetrameric GCN4 coiled coil structures. Proteins: Struct. Funct. Genet, 20, 105-123 (1994).

53. Engel, A.M., Cejka, Z., Lupas, A., Lottspeich, F. \& Baumeister, W. Isolation and Cloning of Ompo, a coiled-coil protein spanning the periplasmic space of the ancestral eubacterium Thermotoga maritima. EMBO J. 11, 4369-4378 (1992)

54. Derouiche, R. et al. TolA central domain interacts with $E$, coli porins. EMBO I. 15 , 6408-6415 (1996).

55. McLachlan, A.D. Coiled-Coil Structure of Murein Lipoprotein. Biochem. Soc Transact. 6, 1353-1354 (1978)

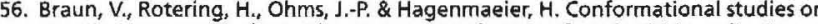
Murein-Lipoprotein from the Outer Membrane of Escherichia coli. Eur. Biochem. 70, 601-610 (1976).

57. Krylov, D. Mikhailenko, I. \& Vinson, C. A thermodynamic scale for leucine zippe stability and dimerization specificity: $\mathrm{e}$ and $\mathrm{g}$ interhelical interactions. $E M B O \mathrm{~J}$. 13, 2849-2861 (1994).

58. Lavigne, P., Sönnichsen, F.D., Kay, C.M. \& Hodges, R.S. Interhelical Salt Bridges Coiled-Coil Stability, and Specificity of Dimerization. Science 271, 1136-1137 (1996).

59. Yang, A.S. \& Honig, B. Electrostatic effects on protein stability. Curr. Op. Struct. Biol. 2, 40-45 (1992).

60. Lumb, K.J. \& Kim, P.S. Measurement of Interhelical Electrostatic Interactions in the GCN4 Leucine Zipper. Science 268, 436-439 (1995).

61. Schülein, K., Schmid, K. \& Benz, R. The sugar-specific outer membrane channel ScrY contains functional characteristics of general diffusion pores and substratespecific porins. Mol. Microbiol. 5, 2233-2241 (1991)

62. Kabsch, W. Automatic Processing of Rotation Diffraction Data from Crystals of Initially Unknown Symmetry and Cell Constants. J. Appl. Cryst. 26, 795-800 (1993)

63. Diederichs, K. A comparison of some heavy-atom refinement and phasing programs. CCP4/ESF-EACBM Newsietters on Protein Crystallography 31, 23-30 (1994). 
64. Kleywegt, G. J. Making the most of your search model. CCP4/ESF-EACBM Newsletter on Protein Crystallography 32, 32-36 (1996)

65. Collaborative Computational Project, Number 4 The CCP4 Suite: Programs for Protein Crystallography. Acta Crystallogr. D50, 760-763 (1994).

66. Cowtan, K. 'dm': An Automated Procedure for Phase Improvement by Density Modification. CCP4IESF-EACBM Newsletter on Protein Crystallography 31, 34-38 (1994).

67. Jones, T. A., Zou, 1.-Y., Cowan, S. W. \& Kjeldgaard, M. Improved methods for building protein models in electron density maps and the location of errors in these models. Acta Crystallogr. A47, 110-119 (1991).

68. Brūnger, A. T. (1992) X-PLOR Version 3.1. A System for X-ray crystallography and NMR (Yale University Press, New Haven; 1992)

69. Brünger, A. T., Krukowski, A. \& Erickson, J. Slow cooling-protocols for crystallographic refinement by simulated annealing. Acta Crystallogr. A46 585-593 (1990)

70. Yeates, T.O. Simple statistics for intensity data from twinned specimens. Act Coystallogr. A44, 142-144 (1988)

71. Gomis-Rüth, F.X. et al. Determination of Hemihedral Twinning and Initial
Structural Analysis of Crystals of the Procarboxypeptidase A Ternary Complex. Acta Crystallogr. D51, 819-823 (1995).

72. Sheldrick, G.M. \& Schneider, T.R. SHELXL: High-Resolution Refinement. Meth Enz. 277, 319-343 (1997).

73. McLachlan, A. D. Gene duplication in the structural evolution of chymotrypsin. J. Mol. Biol. 128, 49-79 (1979)

74. Carson, M. Ribbon Models of Macromolecules, J. Mol. Graphics 5, 103-106 (1987)

75. Kraulis, P. MOLSCRIPT: A program to produce both detailed and schematic plots of protein structures. J.Appl.Crystallogr. 24, 946-950 (1991).

76. Merrit, E.A. \& Bacon, D.J. Raster3D: Photorealistic Molecular Graphics. Meth.Enz. 277, 505-524 (1997)

77. Kabsch, W. \& Sanders, C. Dictionary of protein secondary structure: pattern recognition of hydrogen-bonded and geometrical features. Biopolymers 22 2577-2637 (1983)

78. Nicholls, A., Bharadwaj, R. \& Honig, B. GRASP Graphical Representation and Analysis of Surface Properties. Biophys. J. 64, A166 (1993).

79. Wallace, A.C., Laskowski, R.A. \& Thornton, J.M. LIGPLOT: A program to generate schematic diagrams of protein-ligand interactions. Protein Engng. B, 127-134 (1995) 